\title{
Carbon nanocomposites in the corrosion inhibition
}

\author{
Mikhail Pletnev \\ Chemistry and Chemical Engineering Department, Kalashnikov Izhevsk State Technical University, 426069, Izhevsk, Russia
}

\begin{abstract}
Metal-carbon nanocomposites have been investigated as additives to enhance the protective effect of corrosion inhibitors. Two inhibitors were used as corrosion inhibitors. Inhibitor VNH-L-113 (1morpholinomethylcyclohexylamine) belongs to Schiff bases, which are known as corrosion inhibitors in neutral and acidic media. Inhibitor SNPKh-1004 is often used in the practice of corrosion protection in the oil and gas industry. For research, an electrochemical method was applied using corrosion rate meters of the Monicor type. Neutral aqueous solution and water simulating the corrosive environment of oil fields were chosen as corrosive media. The result shows, that the copper-carbon nanocomposite enhances the protective effect of corrosion inhibitors of various chemical nature in various corrosive environments. The studies carried out make it possible to consider the use of metal-carbon nanocomposites as a promising direction for increasing the effectiveness of inhibitors in the oil and gas industry.
\end{abstract}

\section{Introduction}

The use of corrosion inhibitors is the most important technological method for corrosion protection of oil and gas production equipment and hydrocarbon transport systems. The consumption of inhibitors in the industry is constantly growing [1], therefore, an important factor is to increase the effectiveness of the protective action of inhibitors using various additives. During adsorption at the metal-electrolyte interface of corrosion inhibitors, polymolecularity layers are formed, providing a high protective effect [2].

Nanoparticles of various natures lead to changes in the characteristics of known polymers. For example, the addition of carbon nanoparticles leads to a change in the complex of properties of epoxy resins [3]. Metal/carbon nano-composites have found application in the modification of polymer materials: compounds, adhesives, binders for composite materials based on epoxy resins, polycarbonates, polyvinyl chloride and other polar polymers [4]. We hypothesized that metalcarbon nanocomposites can enter the adsorption layer of the inhibitor and lead to a change in the protective properties. Works on the use of nanoparticles of various natures on the protective effect of inhibitors have already appeared in the literature $[5,6]$. In this work, two inhibitors of different chemical composition were selected as corrosion inhibitors. Inhibitor VNH-L-113 (1-morpholinomethylcyclohexylamine), belongs to Schiff bases, which are known as corrosion inhibitors in neutral [7] and acidic [8] media. Inhibitor SNPKh-1004 is often used in the practice of corrosion protection in the oil and gas industry [9].

\section{Experimental details}

The effect of metal-carbon nanocomposites on the protective effect of the corrosion inhibitor VNKh-L-113 was studied in neutral aqueous solutions of the composition $\mathrm{NaCl} 70 \mathrm{mg} / \mathrm{l}, \mathrm{Na}_{2} \mathrm{SO}_{4} 80 \mathrm{mg} / \mathrm{l}$. To ensure the solubility of the inhibitor, $10 \%$ isopropyl alcohol was added to the electrolyte. Copper-carbon and iron-carbon nanocomposites were introduced into the corrosive medium in the form of a dispersion in isopropyl alcohol in the concentration range $0.005 \%-0.5 \%$ of the inhibitor weight, the concentration of which was constant and amounted to $50 \mathrm{mg} / \mathrm{l}$. To determine the corrosion rate, the corrosion rate indicator Monicor - 1 was used. The exposure time of low-carbon steel samples in a corrosive environment was 90 hours. Description of the experimental procedures in the following experiments are given in the text.

\section{Results and discussion}

It can be seen from the presented results that the studied nanocomposites have different effects on the protective effect of the VNKh-L-113 inhibitor. With an increase in the concentration of the iron-carbon nanocomposite in the studied concentration range, the protective effect of the inhibitor first increases and then decreases (Fig. 1).

* Corresponding author: pletnevm@list.ru 


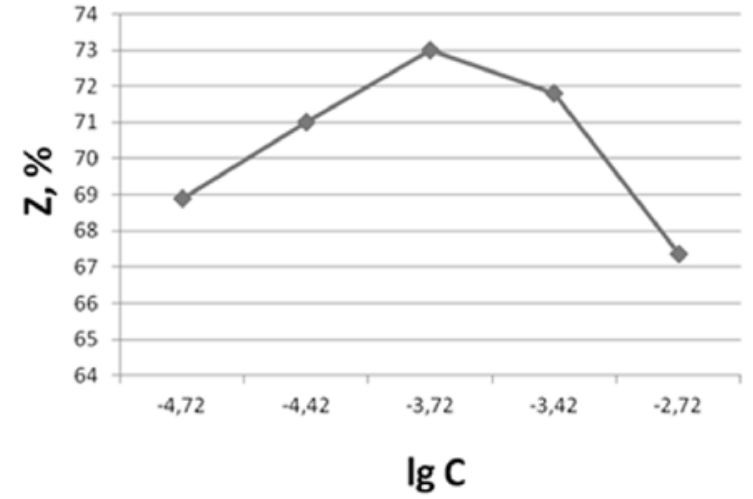

Fig.1. Dependence of the degree of protection of the VNKh-L113 inhibitor on the concentration of the iron-carbon nanocomposite.

Under the same conditions, with an increase in the concentration of the copper-carbon nanocomposite, the protective effect of the inhibitor is increased (Fig.2).

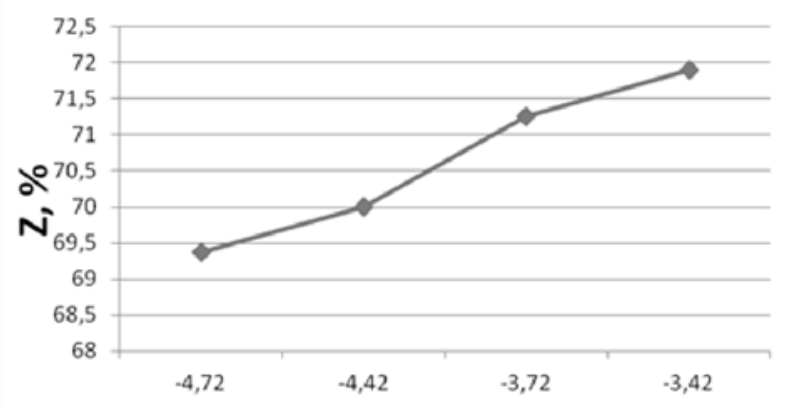

$\lg \mathrm{C}$

Fig.2. Dependence of the degree of protection of the VNKh-L113 inhibitor on the concentration of the copper-carbon nanocomposite.

For further research, a copper-carbon nanocomposite was chosen. The studies were carried out on a model of formation water with a hydrogen sulphide content of 80 $\mathrm{mg} / \mathrm{l}$ by electrochemical and gravimetric methods in accordance with the standard of NK Rosneft [10].

Electrochemical studies were performed using a Monicor-2 corrosion rate indicator. The corrosion inhibitor SNPKh-1004 and the copper-carbon nanocomposite (NC-Cu) in the form of a suspension in isopropyl alcohol were dosed. The total duration of the tests was 6 hours. The measurements were carried out at a working concentration of SNPKh-1004 of $25 \mathrm{mg} / 1$ with a subsequent decrease to $15,10,5 \mathrm{mg} / \mathrm{l}$. The optimal concentrations of the $\mathrm{NC}-\mathrm{Cu}(0.001 ; 0.01 ; 0.1 \mathrm{mg} / \mathrm{l})$ were determined on the basis of the existing experience of their application in other fields and a series of preliminary tests. The results obtained by the electrochemical method are shown in Fig. 3.

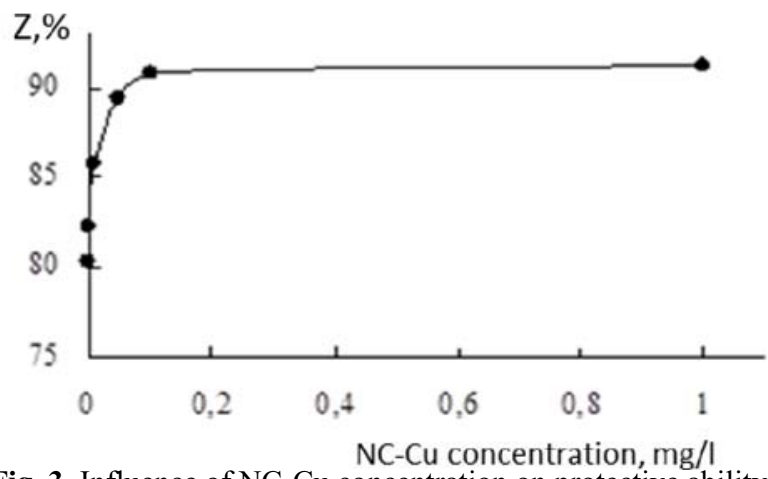

Fig. 3. Influence of $\mathrm{NC}-\mathrm{Cu}$ concentration on protective ability inhibitor SNPKh-1004 (25 mg/1).

Fig. 3 that in a corrosive environment containing 25 $\mathrm{mg} / \mathrm{l} \mathrm{SNPKh}-1004$, with an increase in the concentration of the copper-carbon nanocomposite, the protective effect increases. After that, the effect of the nanocomposite on the protective effect of the inhibitor at lower concentrations was tested.

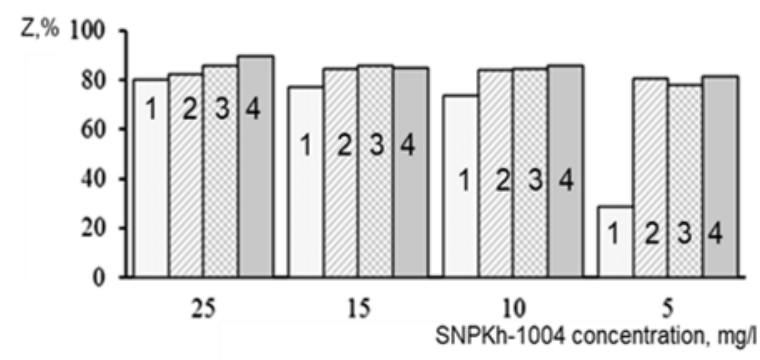

NC-Cu concentration, mg^ $\square 0$-1 $\square 0,001-2 \square 0,01-3 \square 0,1-4$

Fig. 4. Dependence of the degree of protection of the inhibitor on the concentration of SNPKh-1004 and NC-Cu.

A decrease in the concentration of a corrosion inhibitor led to a decrease in its protective effect, while the introduction of a nanocomposite into a corrosive medium increases its efficiency. Regardless of the chosen concentration of the inhibitor, the protective effect remains (Fig. 4).

\section{Conclusions}

Thus, the copper-carbon nanocomposite enhances the protective effect of corrosion inhibitors of various chemical nature in various corrosive environments. The studies carried out make it possible to consider the use of metal-carbon nanocomposites as a promising direction for increasing the effectiveness of inhibitors in the oil and gas industry.

\section{References}

1. Corrosion Inhibitors Market Size, Share \& Trends Report. Report ID: 978-1-68038-210-5

2. M.A. Pletnev, S.M. Reshetnikov, Prot. Met., 38, (2002)

3. S. Bellucci, F. Micciulla, V. M. Levin, Yu. S. Petronyuk, L. A. Chernozatonskii, P. P. Kuzhir, A. 
G. Paddubskaya, J. Macutkevic, M. A. Pletnev, V. Fierro, and A. Celzard, AIP Advances, 5 (2015)

4. V. I. Kodolov, V. V. Trineeva, Nanoscience and Nanoengineering (Apple Academic Press, Waretown, 2019)

5. I. Shevchenko, Bulletin of Science and Practice, 4 (2018)

6. A. Saleh., M. A. Ibrahim, Energy Reports, 5 (2019)

7. A.I. Altsybeeva, M.A. Pletnev, S.M. Reshetnikov and I.B. Shirobokov // Int. J. Corros. Scale Inhib. 8 (2019)

8. P. R. Ammal, M. Prajila, A. Joseph, Egyptian Journal of Petroleum, 27 (2018)

9. N.A. Chirkov, M.A. Gladkikh, V.L.Egorova, A.R.Panteleeva, R.F.Tishankina, S.V. Polovnyak, Oil industry, 5 (2010)

10. Standard of "NK "Rosneft" company № P1-01 SC080 